Stability of a Compact Three-period Stellarator with Guasiaxial Symmetry Features

\author{
W.A. Cooper
}

Published in Physics of Plasmas7(6),

2546 - 2553 (2000) 


\title{
Stability of a compact three-period stellarator with quasiaxial symmetry features
}

\author{
W. A. Cooper* \\ Centre de Recherches en Physique des Plasmas, \\ Association Euratom-Confédération Suisse, \\ Ecole Polytechnique Fédérale de Lausanne, \\ CRPP-PPB, CH-1015 Lausanne, Switzerland
}

March 2, 2000

\begin{abstract}
The ideal magnetohydrodynamic stability properties of a three-field period stellarator with quasiaxial symmetry features proposed by Garabedian and Ku (Phys. Plasmas 6, 645 (1999)) are investigated. The stability limit imposed by local ballooning and Mercier modes allows $\beta \sim 5.3 \%$ to be achieved by tailoring the pressure profile. For this system, the global external kink modes are shown to be stable. To demonstrate this, we have developed a sequence of configurations that varies from the reference system proposed to

${ }^{*}$ E-mail: anthony.cooper@crpp.uhd.epfl.ch
\end{abstract}


the equivalent axisymmetric tokamak. A scan of kink stability reveals that the configuration with a plasma boundary shape that corresponds to $80 \%$ of the basic three-period stellarator and $20 \%$ of the tokamak becomes marginal to global external modes. The normal magnetic field line curvature and the local magnetic shear play crucial roles in the stability properties. The local magnetic shear develops a helical stripe with increasing three-dimensional plasma deformation that inhibits the formation of global structures. The normal magnetic field line curvature, however, becomes locally amplified by the three-dimensional plasma deformation in regions where the local magnetic shear vanishes. As a result, the susceptibility to localized ballooning mode destabilization is enhanced.

52.35.Py, 52.65. $+z, 52.55 . H c$ 


\section{INTRODUCTION}

Stellarator magnetic confinement systems have been considered as attractive options for nuclear fusion reactors because steady state operation without disruptions can be realised in a straightforward manner. Their main drawback, however, has been the poor confinement of the alpha particles that may be insufficient to guarantee self sustained plasma burn. This issue has been successfully resolved with the development of the Wendelstein VII-X concept ${ }^{1}$ that has spurred increased interest in more compact stellarator systems that also benefit from advanced physics properties with respect to energetic particle confinement, magnetohydrodynamic (MHD) stability and plasma exhaust, as well as technical realizability with modular coils. In particular, we focus on devices that are three-dimensional (3D)but in which the magnetic field structure retains symmetry properties similar to those of a tokamak. This is referred to as quasiaxisymmetry. ${ }^{2-6}$

In this paper, we examine a compact three-field period stellarator configuration proposed by Garabedian and $\mathrm{Ku}^{7}$ in which half of the rotational transform is produced by a bootstrap-like plasma current and the other half is supplied externally though 3D plasma shaping effects. They refer to the configuration as a quasiaxially symmetric system. It should be noted, nevertheless, that there is a substantial mirror field component that improves the stability at the expense of neoclassical transport. The predicted energetic particle confinement time is half that of a comparable two-field period device which displays a much higher level of quasiaxisymmetry. Increasing the aspect ratio, however, can improve the symmetry properties of the $B$-field structure. ${ }^{7}$ The $3 \mathrm{D}$ ideal ballooning stability of the device was examined with a pressure profile given by $p(s)=p(0)\left(1-s^{1.5}\right)^{2}$ to yield a $\beta=4 \%$ limit. Here $s$ is a radial variable proportional to the toroidal magnetic flux (and thus the volume) enclosed.

We extend the investigation of the stability properties of this threeperiod configuration in this work. We tailor the pressure profile so that it approaches marginal stability with respect to ballooning modes on 
all the flux surfaces and demonstrate that the $\beta$ limit imposed by the local modes reaches $5.3 \%$. We subsequently examine the global external ideal MHD kink stability properties with respect to periodicity breaking structures and with respect to periodicity preserving structures that destroy the stellarator symmetry. This second class of instability includes the vertical displacement modes. In stellarator systems with finite current, Fu et al. have demonstrated that the external poloidal flux generated through the 3D distortion of the plasma shape exerts a strong stabilizing influence on vertical displacement motion. ${ }^{8}$ We investigate a sequence of configurations where we relax the shaping of the plasma to recover the equivalent axisymmetric tokamak. In this procedure, we fix the pressure profile corresponding to the near marginal ballooning stable profile of the fully $3 \mathrm{D}$ case, but alter the pressure on axis $\mathrm{p}(0)$ to maintain $\beta \simeq 5.3 \%$. We also fix the prescribed toroidal plasma current profile but vary the total toroidal plasma current to maintain the maximimum value of the rotational transform in the plasma at $\iota_{\max } \simeq 0.484$. The shape of the rotational transform profile varies somewhat in the sequence that encompasses the 3D compact stellarator proposed in Ref. 7 and the corresponding equivalent tokamak.

The conditions of marginal stability of the compact three-period stellarator with quasiaxial symmetry features with respect to local ballooning modes is discussed in Sec. II.In Sec. III, a sequence of configurations that varies from this compact stellarator to the equivalent axisymmetric tokamak is developed. The global ideal MHD stability properties of the sequence is examined in Sec. IV. The impact of the magnetic field line curvature and the local magnetic shear on global and local stability is analyzed in Sec. V, followed by the conclusions in Sec.VI.

\section{CONDITIONS OF MARGINAL LOCAL STABILITY}

We apply the fixed boundary version of the VMEC code to compute 
3D equilibria with nested magnetic flux surfaces. ${ }^{9}$ The inputs required for this code are the Fourier spectrum of the plasma boundary surface reported in Ref. 7 , the pressure profile and the toroidal plasma current profile. A hollow toroidal plasma current with a finite edge gradient that models the bootstrap effect is prescribed as

$$
2 \pi J^{\prime}(s)=2 \pi J(1)\left[0.9(1-s)+20 s^{3}(1-s)\right] / 1.45,
$$

where the magnitude of the current is specified by $2 \pi J(1)$. A typical profile is presented in Fig.1. The pressure profile is initially prescribed as

$$
p(s)=p(0)\left(1-s^{2}\right)^{2}
$$

and the volume average $\beta$ is defined as

$$
\beta=\frac{2 \mu_{0} \int d^{3} x p}{\int d^{3} x B^{2}} .
$$

The local modules of the 3D TERPSICHORE stability code described in Ref. 10 are used to evaluate the ballooning eigenvalue and the Mercier criterion on each flux surface. The pressure profile is subsequently adjusted to satisfy the ballooning stability criteria on each flux surface. The initial and marginally stable $p(s)$ profiles and the corresponding rotational transform profiles are displayed in Fig. 2. The Mercier criteria for the ballooning stable profiles also predict stability except on a few isolated surfaces located close to mode rational contours. We interpret this as potential magnetic island formation rather than a strict stability limit.

\section{SEQUENCE OF CONFIGURATIONS}

The mod- $B^{2}$ distribution for five configurations of the sequence is shown in Fig. 3 on three different cross sections encompassing the axisymmetric and the fully $3 \mathrm{D}$ systems under study. The reference case ballooning marginal $p(s)$ and $2 \pi J^{\prime}(s)$ profiles are fixed in this sequence while $p(0)$ and $2 \pi J(1)$ are altered to $\operatorname{keep} \beta \simeq 5.3 \%$ and $\iota_{\max } \simeq 0.484$, 
respectively. In Fig. 4, the normalized toroidal plasma current $2 \pi J(1)$ is plotted as a function of the plasma shaping parameter $T$. It shows that the reference case has half the toroidal current of the equivalent tokamak. This underlies the statement that about half the rotational transform in the reference case is supplied by the plasma current and the other half by the 3D plasma shaping effect. The $\iota(s)$ profiles for cases with several values of $T$ are also displayed to illustrate that there is a small variation from case to case. A Fourier analysis of $B^{2}$ in Boozer magnetic coordinates ${ }^{11}$ for a nearly axisymmetric configuration obtained with $T=1 / 4$ and for the $T=1$ reference case is shown in Fig. 5. Though the leading component is axisymmetric for $T=1$, there is a significant mirror term contribution also.

\section{GLOBAL EXTERNAL STABILITY}

The global modules of the 3D TERPSICHORE code $^{12}$ are applied to explore the external kink stability properties of the sequence of configurations described in Sec. III. An axisymmetric conducting wall of aspect ratio unity is prescribed to undertake the external mode study as illustrated for the $T=1$ and $T=1 / 4$ configurations in Fig. 6. The wall is sufficiently far from the plasma-vacuum interface to simulate the conditions of a wall at infinity. We concentrate in this work on three global mode structures, namely, the periodicity breaking $m / n=3 / 1$ external kink, the periodicity preserving $m / n=7 / 3$ external kink which breaks the stellarator symmetry and the $m / n=1 / 0$ vertical displacement motion. The vertical $m / n=1 / 0$ instability is actually coupled to the $m / n=7 / 3$ external kink (it is a member of the same family ${ }^{13,14}$ ), but which component dominates depends on the plasma shaping. The global unstable eigenvalues as a function of the plasma shaping parameter $T$ are displayed in Fig. 7. At $\beta \simeq 5 / 3 \%$, the $m / n=7 / 3$ and $m / n=3 / 1$ external kink modes yield similar marginal stability conditions that correspond to the configuration obtained with $T \simeq 0.8$. The vertical displacement mode dominates the 
$m / n=7 / 3$ external kink for $T<0.4$ and predicts marginal stability for $T \simeq 0.6$. The leading Fourier amplitudes of the radial component of the perturbed displacement vector as a function of the radial variable $s$ are shown in Fig. 8 for the $T=0.25$ configuration. The vertical instability has an eigenvalue $\lambda=-0.007$, making it the most unstable member of its family. It should be noted, nevertheless, that this mode still retains a nonnegligible helical $m / n=7 / 3$ contribution. The second most unstable member of the family is dominated by $n=3$ components (with $m=7$ the largest) and has an eigenvalue $\lambda=-0.0047$. For the $T=0.79$ configuration, the leading Fourier amplitudes of the radial component of the perturbed displacement vector versus $s$ for the periodicity breaking $m / n=3 / 1$ and the stellarator symmetry breaking $m / n=7 / 3$ external kinks are displayed in Fig. 9 . This configuration is nearly marginal as manifested by the magnitude of the eigenvalues $\lambda=-0.0001$ and $\lambda=-0.0002$ associated with the $m / n=3 / 1$ and $m / n=7 / 3$ dominated structures, respectively. The perturbed pressure distribution $\delta p\left(\delta p=-p^{\prime}(s) \boldsymbol{\xi} \cdot \nabla \mathrm{s}\right.$, where $\boldsymbol{\xi}$ is the perturbed displacement vector) for the stellarator symmetry breaking $m / n=1 / 0$ vertical displacement motion that constitutes the most unstable mode of the $n=0$ family $(\lambda=-0.007)$ for the $T=0.25$ configuration is compared in the top row of Fig. 10 with the second most unstable mode of this family $(\lambda=-0.0047)$ which is an external kink structure dominated by a $m / n=7 / 3$ component. Also compared is the $\delta p$ distribution from a periodicity breaking instability dominated by a $m / n=3 / 1$ structure with that of a stellarator symmetry instability dominated by a $m / n=7 / 3$ structure that preserves the periodicity of the underlying equilibrium state for the near marginally stable system obtained with $T=0.79$. This is displayed in the upper-middle row of Fig. 10.

\section{DISCUSSION}

To attempt to understand the physical mechanisms that govern 
the stability properties of compact stellarator systems with quasiaxial symmetry features, we analyze the interaction of the pressure gradient with the normal magnetic field line curvature $2 p^{\prime}(s) \sqrt{g} \kappa \cdot \nabla s$ and the local magnetic shear term $\sqrt{g} S^{15,16}$ which constitute fundamental destabilizing and stabilizing contributions, respectively, for MHD instabilities. The Jacobian of the transformation to Boozer coordinates is denoted by $\sqrt{g}$. The normal curvature and the local shear distributions on a toroidal magnetic flux surface near mid-volume for the nearly axisymmetric configuration obtained with $T=1 / 4$ is compared with that of the reference case given by $T=1$ in the lower half of Fig. 10. On the inside edge of the flux surface, the $2 p^{\prime}(s) \sqrt{g} \kappa \cdot \nabla s$ distribution is fairly uniform, positive and shows a weak toroidal modulation. On the outside edge of the torus, on the other hand, this distribution evolves from quasi uniform with a value around -1 for the $T=1 / 4$ case to strongly modulated toroidally with a value that reaches -2 for the $T=1$ case as seen in the lower-middle row of Fig. 10. The outside edge of the up-down symmetric cross section that is bean shaped aligns with the most negative and thus destabilizing values of $2 p^{\prime}(s) \sqrt{g} \kappa \cdot \nabla s$. The toroidal average, however, is comparable in both cases. For the comparison of the corresponding local shear distributions shown in the bottom row of Fig. 10, we concentrate on the outside edge of the torus where the curvature is destabilizing. We see that the $\sqrt{g} S$ distribution for the $T=1 / 4$ case displays a weak helical modulation with values oscillating between 0 and -0.3 . For the $T=1$ reference system, the $\sqrt{g} S$ distribution develops a sharp helical stripe which varies between -0.5 and -1.5 in magnitude. In between the stripes, there is a broad domain where the local magnetic shear becomes nearly vanishing. From the perusal of the structures of $2 p^{\prime}(s) \sqrt{g} \kappa \cdot \nabla s$ and $\sqrt{g} S$ for the weakly 3D $T=1 / 4$ and the reference $T=1$ configurations, one can anticipate that the large negative helical stripe of $\sqrt{g} S$ would inhibit the formation of instability structures that extend beyond one equilibrium field period. This is confirmed by the results presented in Fig. 7. Furthermore, mode structures that can concentrate within the region in which the 3D deformation of the plasma on the one hand enhances the negative value of $2 p^{\prime}(s) \sqrt{g} \kappa \cdot \nabla s$ 
and on the other drives $\sqrt{g} S$ to vanish can become increasingly destabilized. As verification, we plot the ballooning eigenvalue profiles for different configurations of the sequence considered in Fig. 11. The localized ballooning modes, which are nearly marginal for the reference $T=1$ system, become strongly stabilized in the outer half of the plasma volume as the $3 \mathrm{D}$ shaping is relaxed at fixed $\beta=5.3 \%$.

\section{CONCLUSIONS}

We have investigated the local and global ideal MHD stability properties of a compact three-field period stellarator system with quasiaxial symmetry features proposed by Garabedian and $\mathrm{Ku}^{7}$ Localized ballooning instabilities predict a limiting $\beta \simeq 5.3 \%$ when the pressure profile is tailored to approach marginal conditions nearly uniformly on all the flux surfaces. A sequence of configurations has been developed by linearly varying the magnitude of the nonaxisymmetric components of the Fourier spectrum the describes the plasma boundary shape. The global external kink stability investigation has revealed that the axisymmetric limit of this sequence is unstable and that the helical deformation of the plasma has a stabilizing impact. The configuration for which the magnitude of the nonaxisymmetric components of the boundary spectrum corresponds to $80 \%$ of the basic system described in Ref. 7 becomes marginal with respect to external kinks. For vertical displacement motion stability, the corresponding shaping factor is $60 \%$.

The helical deformation of the plasma deteriorates the stability with respect to local ballooning modes but improves it with respect to global external kinks. The 3D shaping of the plasma causes the interaction of the pressure gradient with the normal magnetic field line curvature to increase in toroidally local regions on the outside

edge of the torus which coincide with vanishing conditions of the local magnetic shear. On the other hand, the normal cuvature on average is unchanged around the torus while the local magnetic shear develops 
a helical stripe of significant magnitude that inhibits the formation of instability structures that extend beyond one field period. This accounts for the seemingly conflicting effects of helical plasma shaping on local and global ideal MHD stability predictions in 3D magnetic confinement systems.

\section{ACKNOWLEDGMENTS}

This research was partially sponsored by the Fonds National Suisse de la Recherche Scientifique and by Euratom. We thank Dr. S.P. Hirshman for use of the VMEC equilibrium code. The numerical calculations have been performed on the NEC-SX4 computer at the Centro Svizzero di Calcolo Scientifico in Manno, Switzerland. 
${ }^{1}$ G. Grieger, C.D. Beidler, H. Maassberg, E. Harmeyer, F. Herrnegger, J. Junker, J. Kisslinger, W. Lotz, P. Merkel, J. Nührenberg, F. Rau, J. Sapper, A. Schlüter, F. Sardei and H. Wobig, in Plasma Physics and Controlled Nuclear Fusion Research 1990 (International Atomic Energy Agency, 1991), Vol. 3, p. 525.

${ }^{2}$ J. Nührenberg, W. Lotz and S. Gori, Quasi-axisymmetric Tokamaks, in Proc. Joint Varenna-Lausanne Int. Workshop on Theory of Fusion Plasmas, E. Sindoni, F. Troyon and J. Vaclavik, eds. (Soc. Ital. Fisica - Editrice Compositori, Bologna, 1994) pp. 3-12.

${ }^{3}$ P.R. Garabedian, Phys. Plasmas 3, 2483 (1996).

${ }^{4}$ N. Nakajima, M. Yokoyama, M. Okamoto and J. Nührenberg, Plasma Phys. Reports 23, 460 (1997).

${ }^{5}$ S. Okamura, K. Matsuoka, M. Fujiwara, M. Drevlak, P. Merkel and J. Nührenberg, J. Plasma and Fusion Res. SERIES 1, 164 (1998).

${ }^{6}$ A. Reiman, G. Fu, S. Hirshman, L. Ku, D. Monticello, H. Mynick, M. Redi, D. Spong, M. Zarnstorff, B. Blackwell, A. Boozer, A. Brooks, W.A. Cooper, M. Drevlak, R. Goldston, J. Harris, M. Isaev, C. Kessel, Z. Lin, J.F. Lyon, P. Merkel, M. Mikhailov, W. Miner, N. Nakajima, G. Neilson, C. Nührenberg, M. Okamoto, N. Pomphrey, W. Reiersen, R. Sanchez, J. Schmidt, A. Subbotin, P. Valanju, K.Y. Watanabe and R. White, Plasma Phys. Control. Fusion 41, B273 (1999).

${ }^{7}$ P. Garabedian and L.P. Ku, Phys. Plasmas 6, 645 (1999).

${ }^{8}$ G.Y. Fu, L.P. Ku, W.A. Cooper, S.P. Hirshman, D.A. Monticello, M.H. Redi, A. Reiman, R. Sanchez and D.A. Spong, submitted to Phys. Plasmas (2000).

${ }^{9}$ S.P. Hirshman and O. Betancourt, J. Comput. Phys. 96, 99 (1991).

${ }^{10}$ W.A. Cooper, Plasma Phys. Contr. Fusion 34, 1011 (1992).

${ }^{11}$ A.H. Boozer, Phys. Fluids 23, 904 (1980).

${ }^{12}$ D.V. Anderson, W.A. Cooper, R. Gruber, S. Merazzi, and U. Schwenn, Int. J. Supercomp. Appl. 4, 34 (1990).

${ }^{13}$ W.A. Cooper, G.Y. Fu, R. Gruber, S. Merazzi, U. Schwenn and D.V. Anderson, Global external ideal magnetohydrodynamic instabilities in three-dimensional plasmas, in Proc. Joint Varenna-Lausanne Int. Workshop on Theory of Fusion Plasmas, E. Sindoni, F. Troyon and J. Vaclavik, eds. (Soc. Ital. Fisica - Editrice Compositori, Bologna, 1990) pp. 655-665.

${ }^{14}$ C. Nührenberg, Phys. Plasmas 3, 2401 (1996).

${ }^{15}$ J.M. Greene and J.L. Johnson, Plasma Phys. 10, 729 (1968).

${ }^{16}$ J.M. Greene and M.S. Chance, Nucl. Fusion 21, 453 (1981). 
Table 1: Fourier decomposition of the boundary of the configuration sequence. $T$ varies from 0 to 1 . The poloidal mode number is $m$, the toroidal mode number is $n$ and $L=3$ is the number of field periods of the system.

\begin{tabular}{lrll}
\hline \hline & & & \\
& $n / L$ & $R_{m n}$ & $Z_{m n}$ \\
\hline 0 & 0 & +3.50 & +0.00 \\
0 & 1 & $+0.10 T$ & $+0.10 T$ \\
0 & 2 & $-0.01 T$ & $+0.01 T$ \\
1 & -2 & $-0.02 T$ & $-0.02 T$ \\
1 & -1 & $+0.02 T$ & +0.00 \\
1 & 0 & +0.70 & +1.30 \\
1 & 1 & $-0.30 T$ & $+0.30 T$ \\
1 & 2 & $+0.04 T$ & $-0.04 T$ \\
1 & 3 & $+0.02 T$ & $-0.02 T$ \\
2 & 0 & +0.18 & +0.00 \\
2 & 1 & $+0.12 T$ & $+0.18 T$ \\
2 & 2 & $+0.06 T$ & $-0.06 T$ \\
3 & -1 & $+0.01 T$ & $-0.01 T$ \\
3 & 0 & -0.02 & +0.02 \\
3 & 1 & $+0.02 T$ & $-0.02 T$ \\
3 & 3 & $-0.02 T$ & $+0.02 T$ \\
\hline \hline
\end{tabular}




\section{Figures}

FIG.1. The prescribed toroidal plasma current profile.

FIG.2. The initial pressure profile $(\diamond)$, the pressure profile adjusted to satisfy ballooning stability $(o)$ (top) and the corresponding rotational transform $\iota$ profiles (bottom).

FIG.3. The mod- $B^{2}$ distribution in Boozer magnetic coordinates for the configurations with $T=0, T=0.25, T=0.5, T=0.79$ and $T=1$ from top to bottom, respectively, on cross sections with toroidal angle $\phi=0$ (left) at the beginning of a field period, with $\phi=\pi / 6$ (middle) at $1 / 4$ of a period and with $\phi=\pi / 3$ (right) at mid-period at $\beta \simeq 5.3 \%$.

FIG.4. The normalized toroidal plasma current as a function of $T$ (top) and the $\iota$ profiles for $T=0, T=0.5, T=0.79$ and $T=1$ (bottom).

FIG.5. The leading Fourier amplitudes of $B^{2}$ for a nearly axisymmetric configuration with $T=1 / 4$ (top) and for the reference system obtained with $T=1$ (bottom). For the $B_{00}$ component, its value on axis has been subtracted. The reference case has a dominant axisymmteric and a significant mirror contribution.

FIG.6. The plasma boundary shape (solid line) and the prescribed axisymmetric conducting wall (dotted line) for the reference system obtained with $T=1$ (top) and for the more nearly axisymmetric cross section obtained with $T=1 / 4$ (bottom) on cross sections at the beginning of a field period (right), at $1 / 4$ of a field period (middle) and at mid-period (right).

FIG.7. The global external kink eigenvalues as a function of the plasma shaping parameter $T$ for structures dominated by the periodicity breaking $m / n=3 / 1$ component, by the periodicity preserving but stellarator symmetry breaking $m / n=7 / 3$ component and by the vertical displacement $m / n=1 / 0$ motion.

FIG.8. The leading Fourier amplitudes of the radial component of the displacement vector as a function of the radial variable $s$ that correspond to stellarator symmetry breaking structures dominated by a $m / n=1 / 0$ vertical motion (top) and by a $m / n=7 / 3$ external kink (bottom) for the configuration with $T=0.25$.

FIG.9. The leading Fourier amplitudes of the radial component of the displacement vector as a function of the radial variable $s$ corresponding to periodicity breaking $m / n=3 / 1$ dominant structures (top) and to stellarator symmetry breaking $m / n=7 / 3$ external kink structures (bottom) for the configuration with $T=0.79$. 
FIG.10. The perturbed pressure distribution on a toroidal magnetic surface near the edge of the plasma for a nearly axisymmetric system at $\beta=5.3 \%$ obtained with $T=0.25$ (top row) from unstable stellarator symmetry breaking $m / n=$ $1 / 0$ vertical motion structures (left) and $m / n=7 / 3$ external kink structures (right). The corresponding distribution for the nearly marginal case obtained with $T=0.79$ (upper-middle row) from periodicity breaking $m / n=3 / 1$ external kink structures (left) and stellarator symmetry breaking $m / n=7 / 3$ external kink structures (right). The $2 p^{\prime}(s) \sqrt{g} \kappa \cdot \nabla s$ (lower-middle row) and $\sqrt{g} S$ (bottom row) distributions on a toroidal toroidal magnetic flux surface near mid-volume at $\beta=5.3 \%$ for a nearly axisymmetric configuration obtained with $T=1 / 4$ (left) and for the $T=1$ reference case (right).

FIG.11. The ballooning eigenvalue profiles with a pressure profile adjusted to satisfy marginal stability conditions for the reference configuration obtained with $T=$ 1 and for the configurations with $T=0.79, T=0.5, T=0.25$ and $T=0$. 


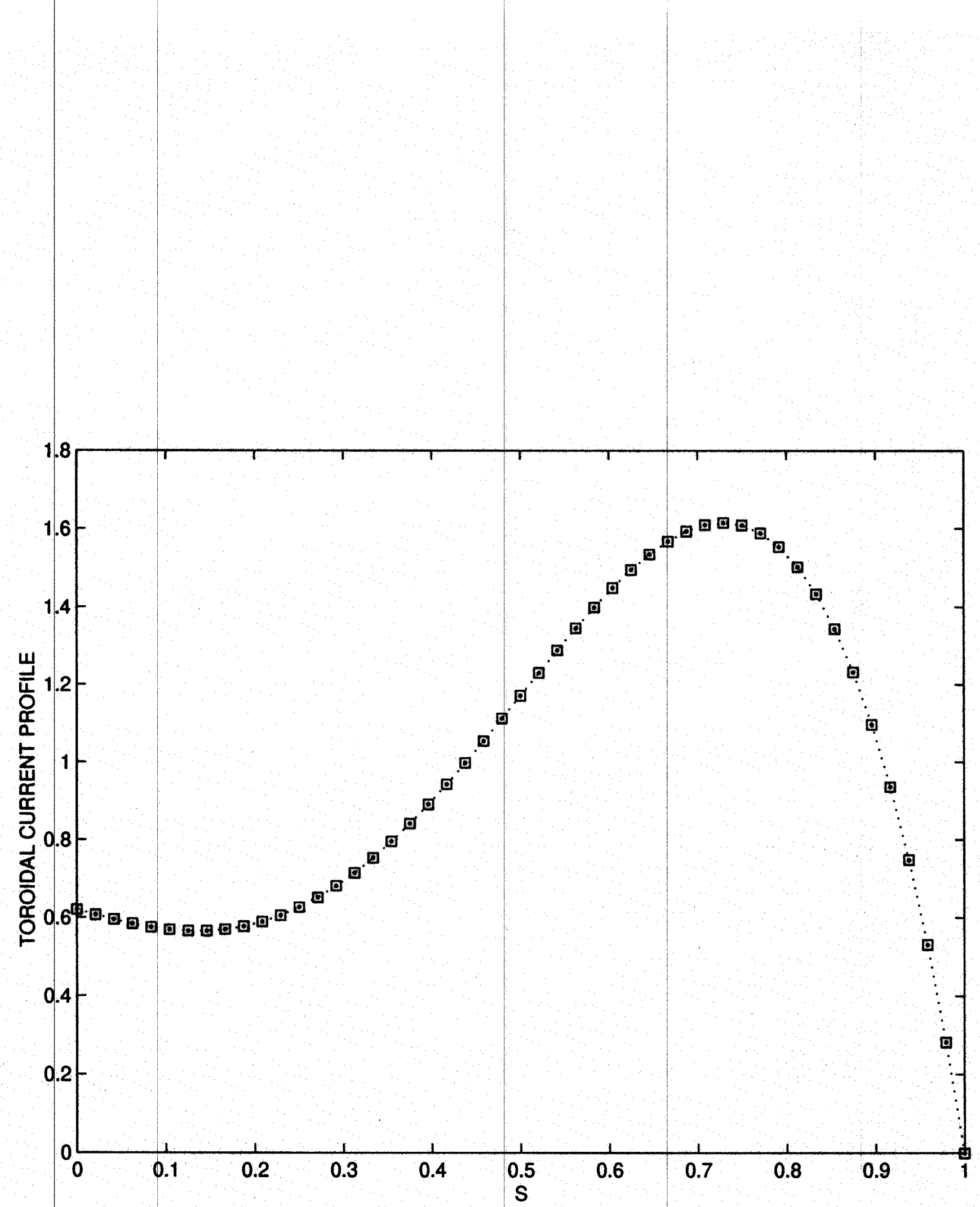

Figure 1: 

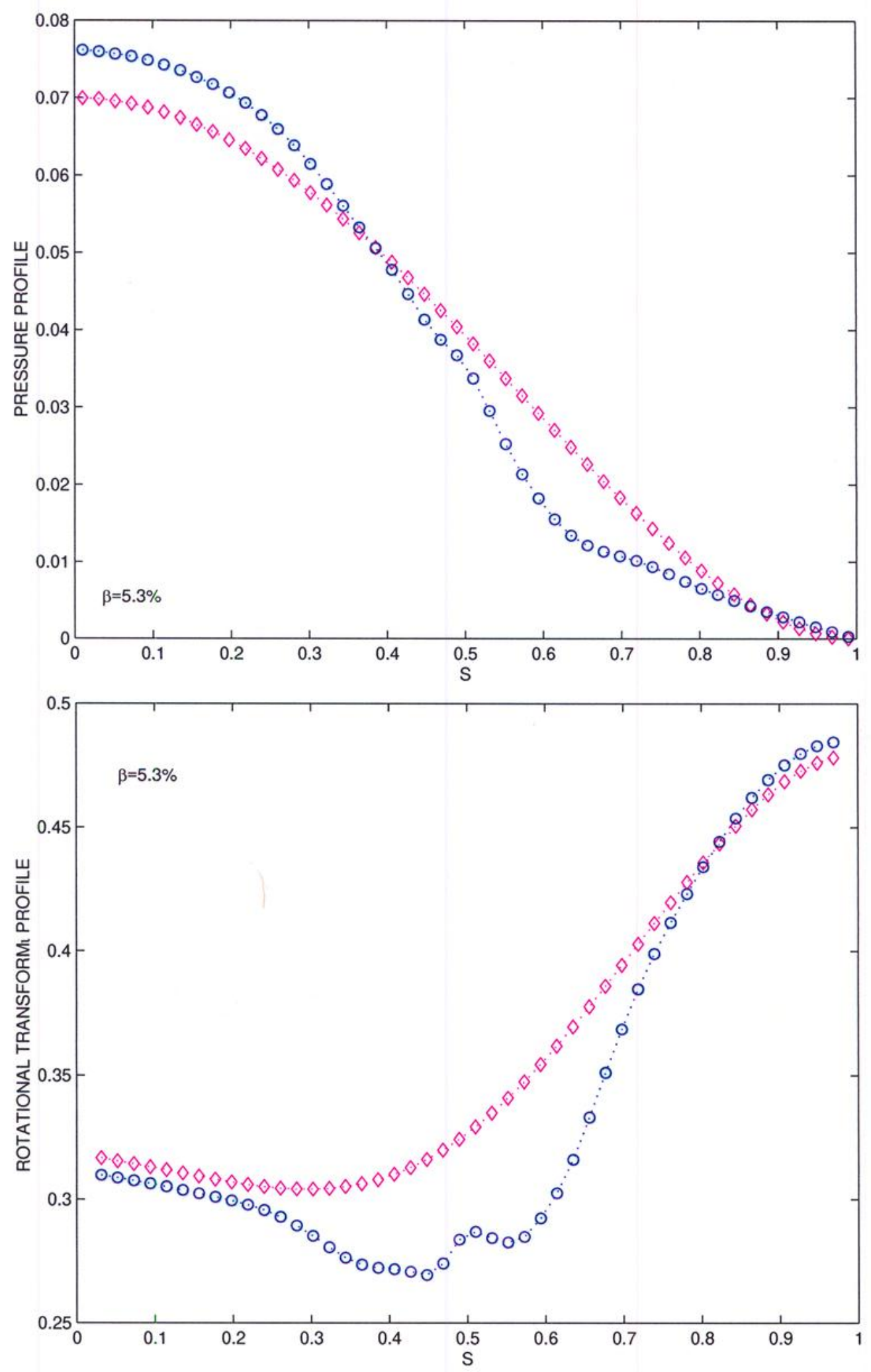

Figure 2: 

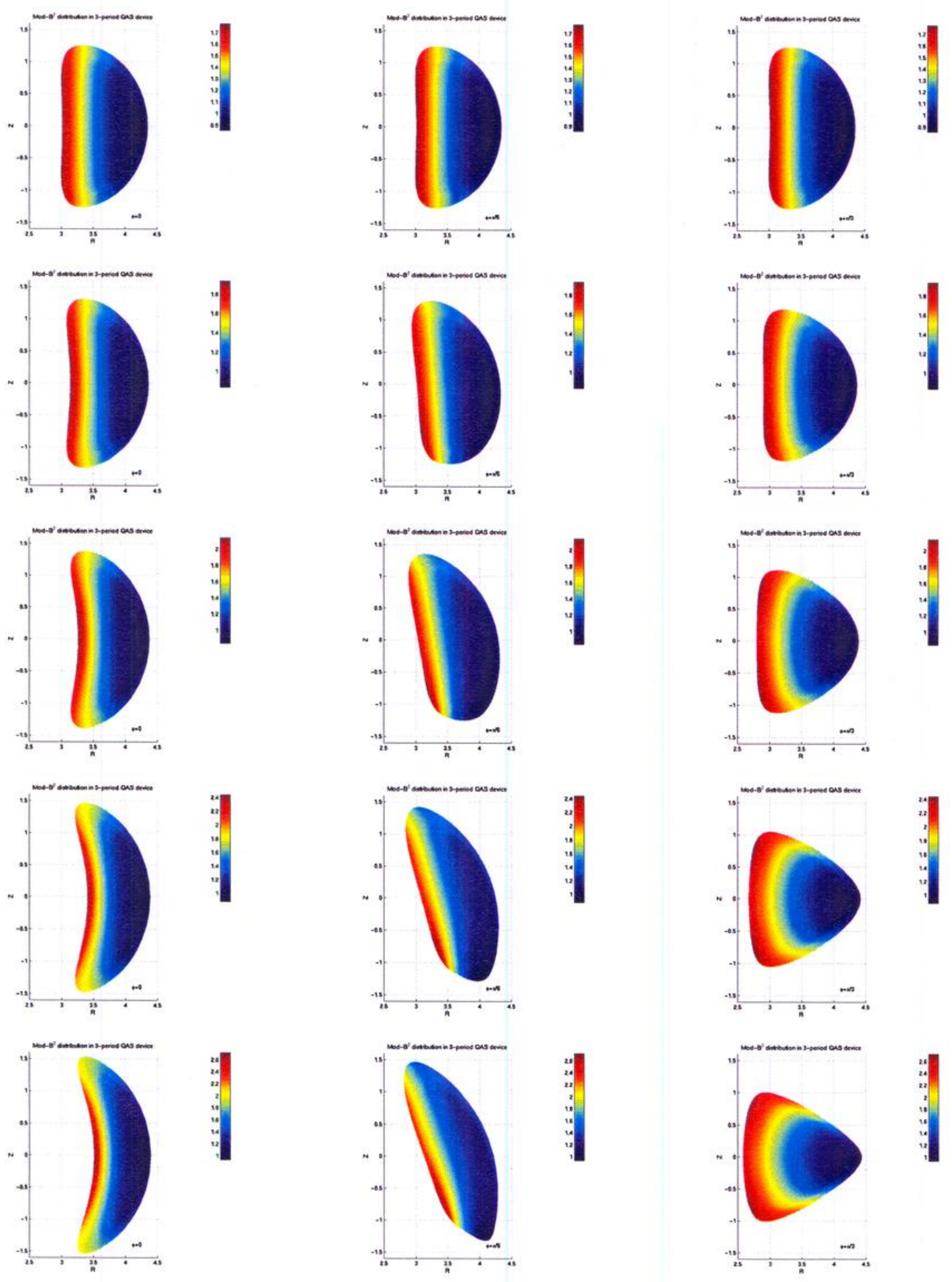

Figure 3: 

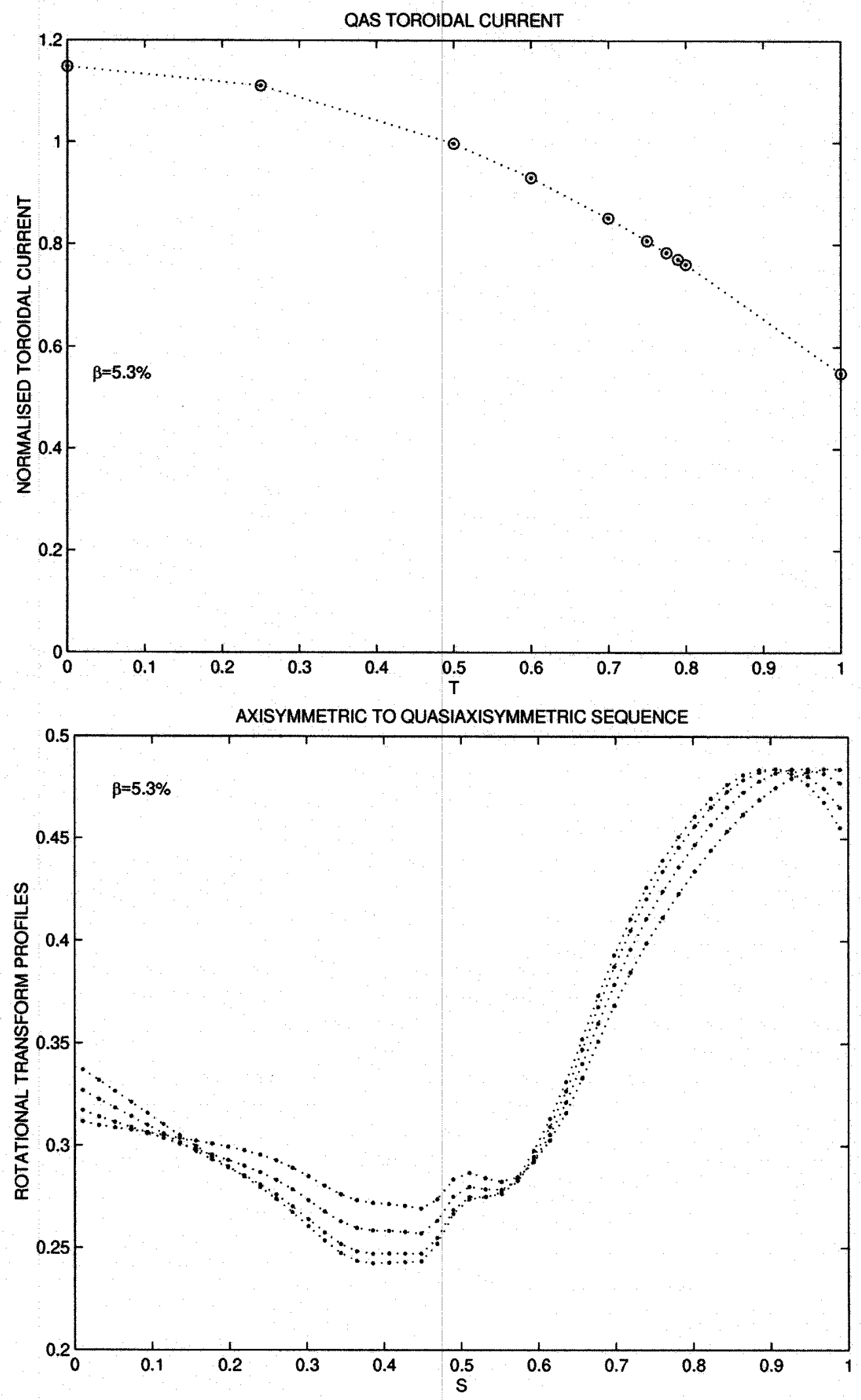

Figure 4: 

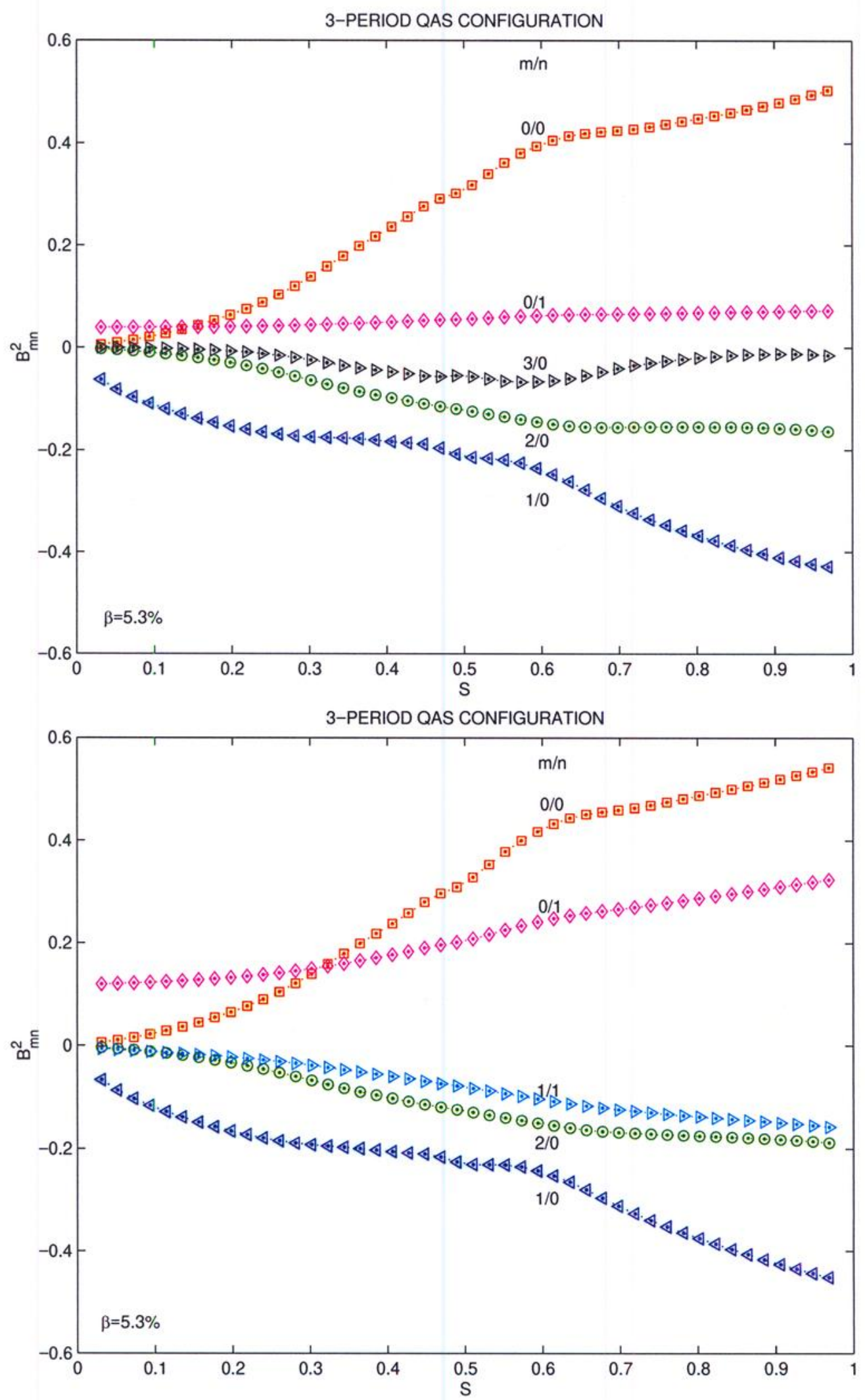

Figure 5: 

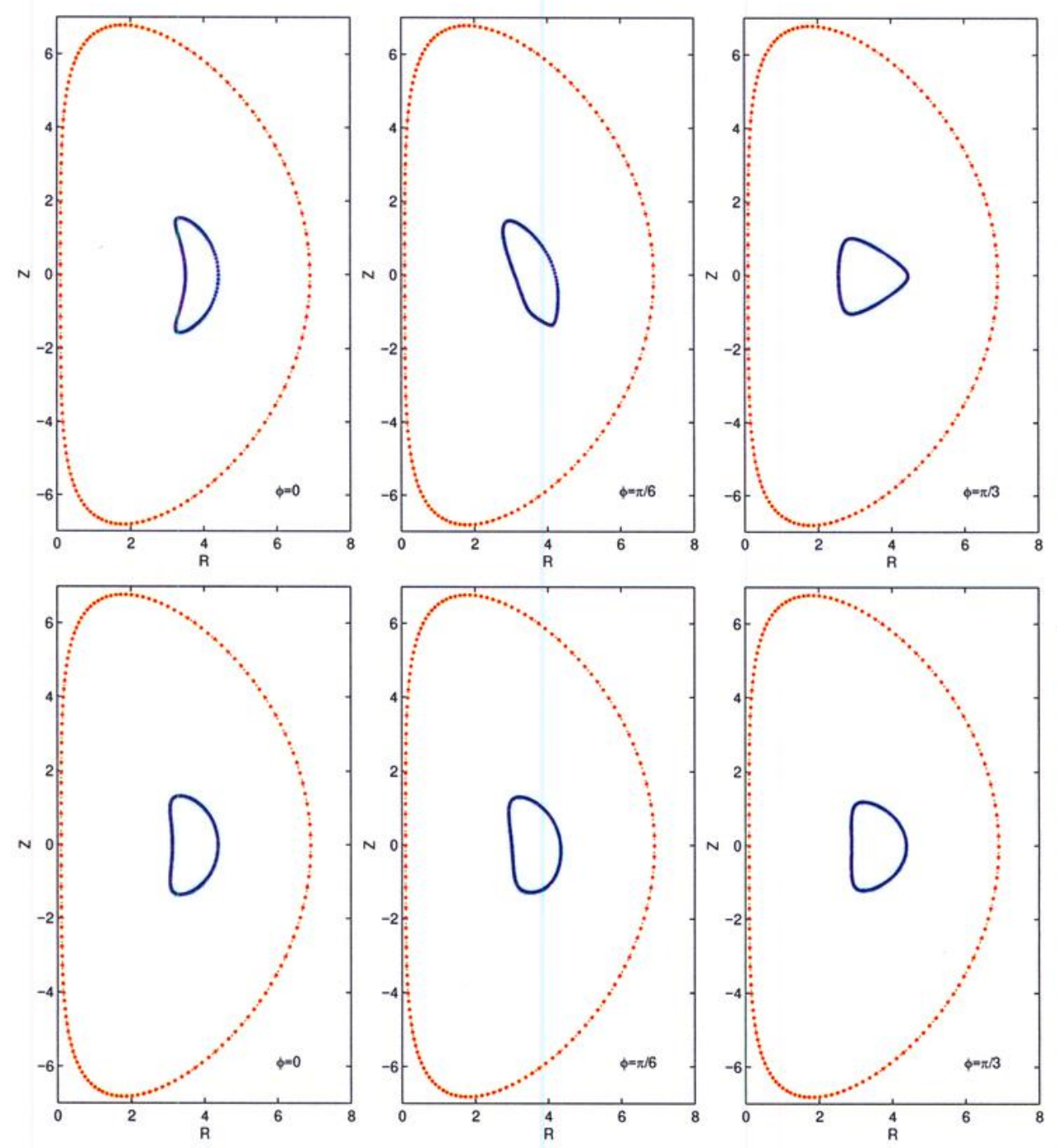

Figure 6:

20 


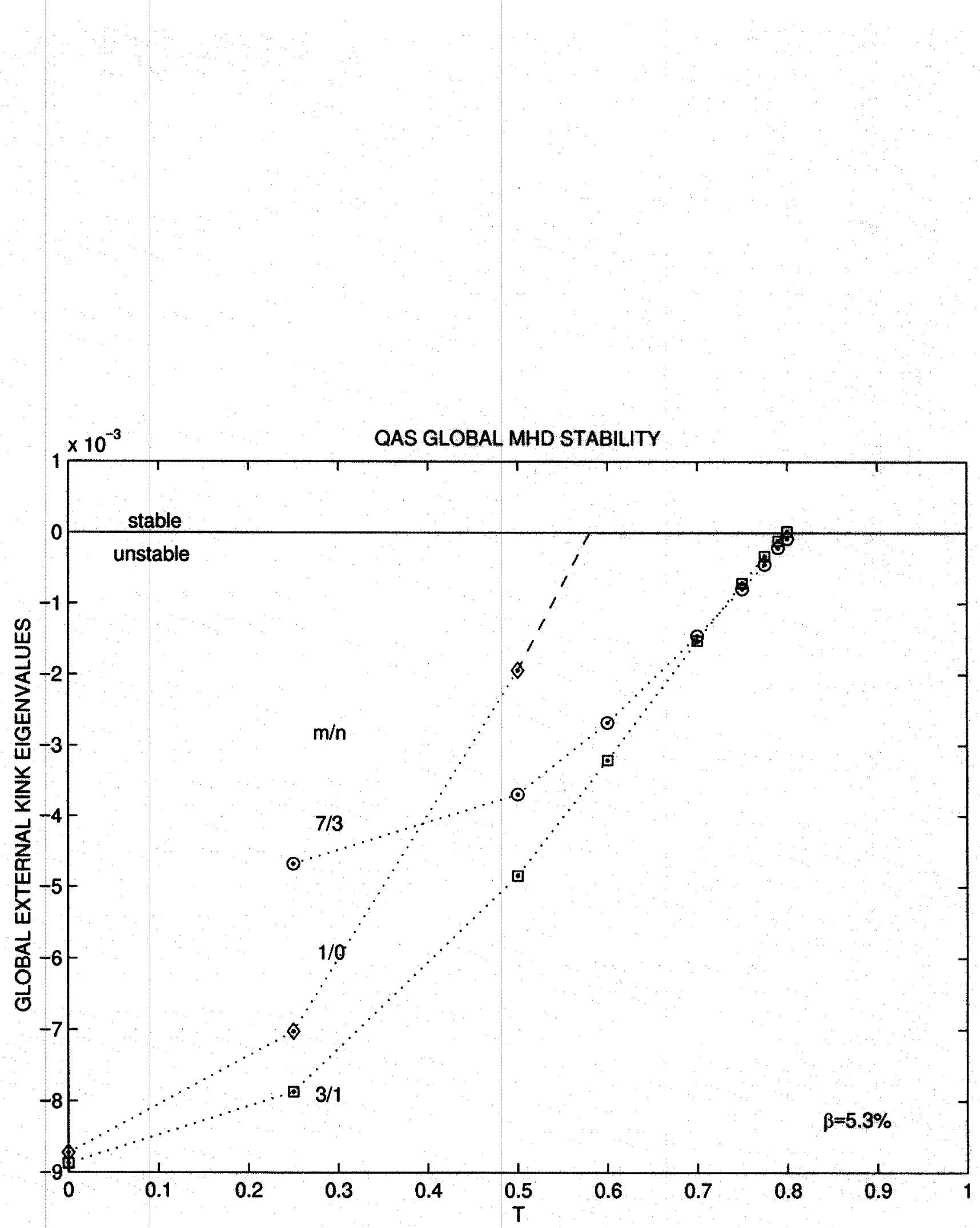

Figure 7: 


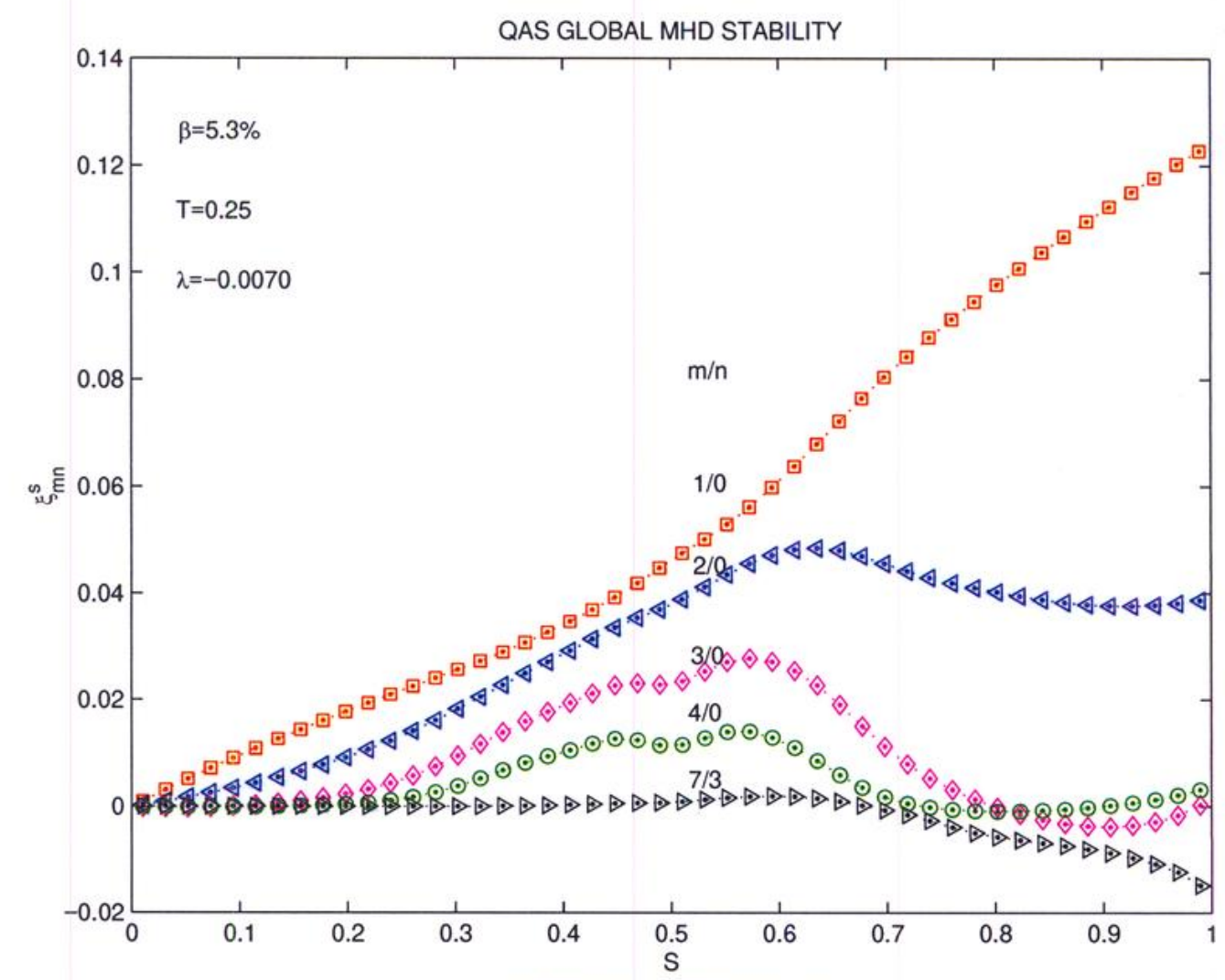

QAS GLOBAL MHD STABILITY

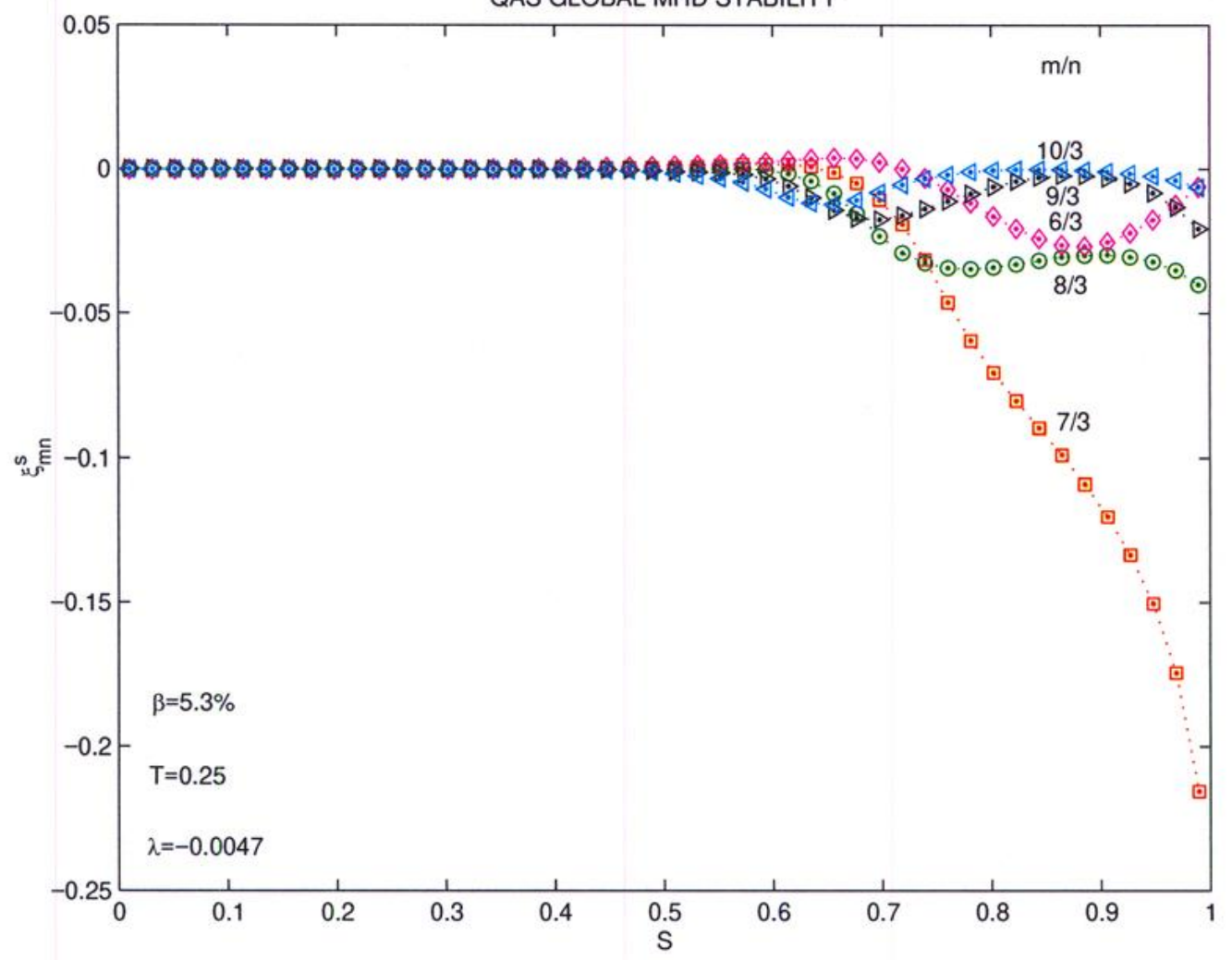

Figure 8: 

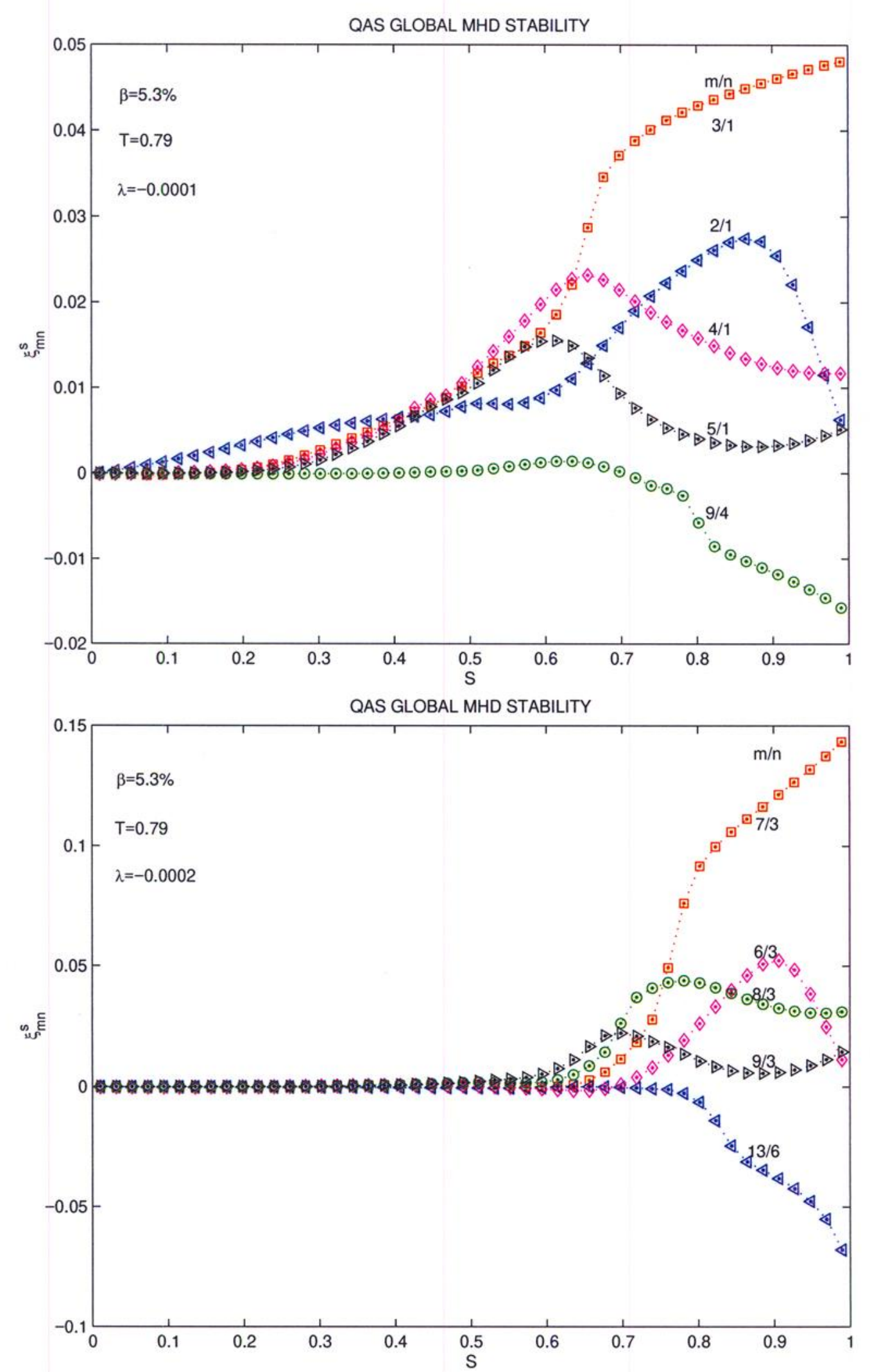

Figure 9: 

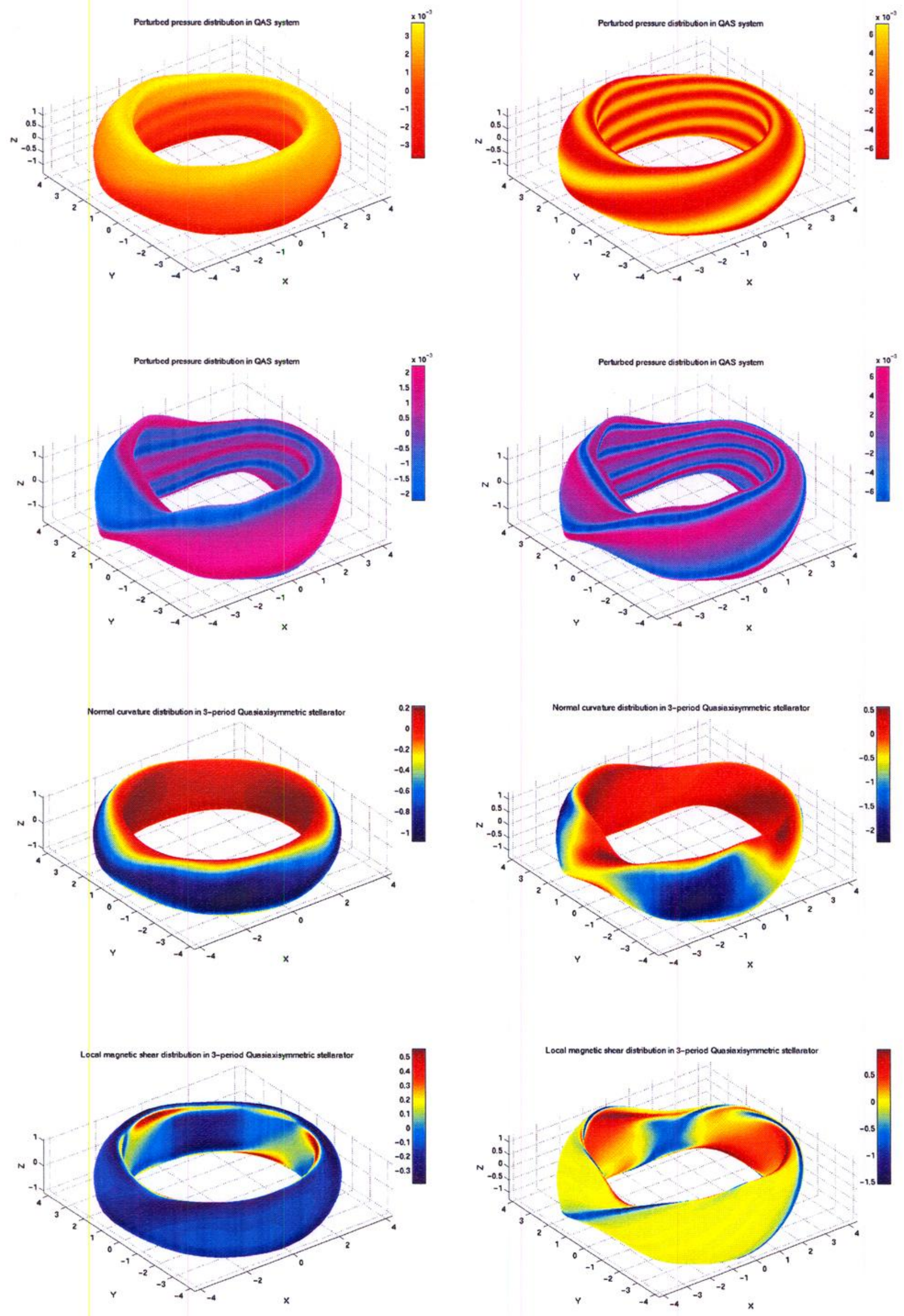

Figure 10: 


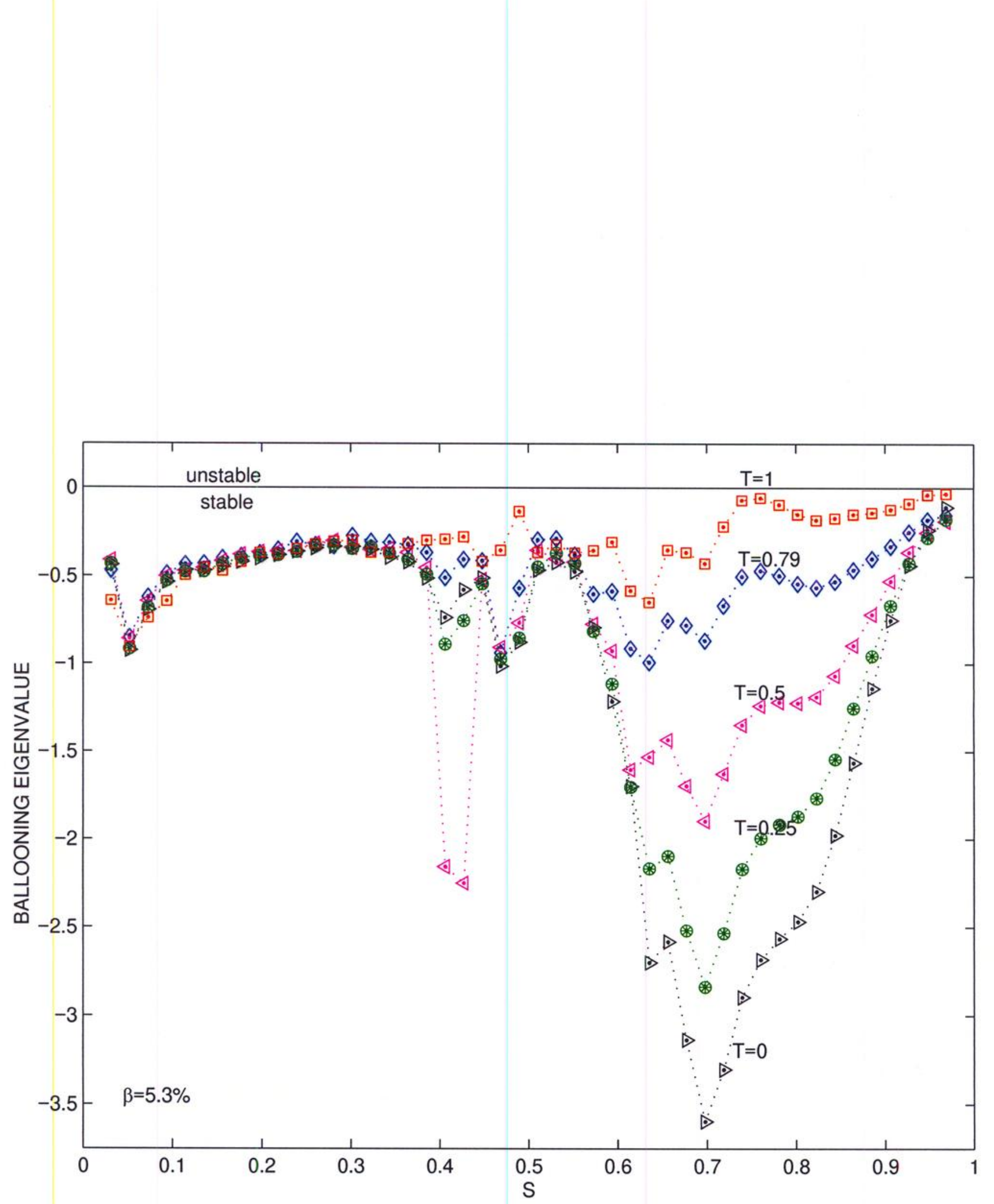

Figure 11: 